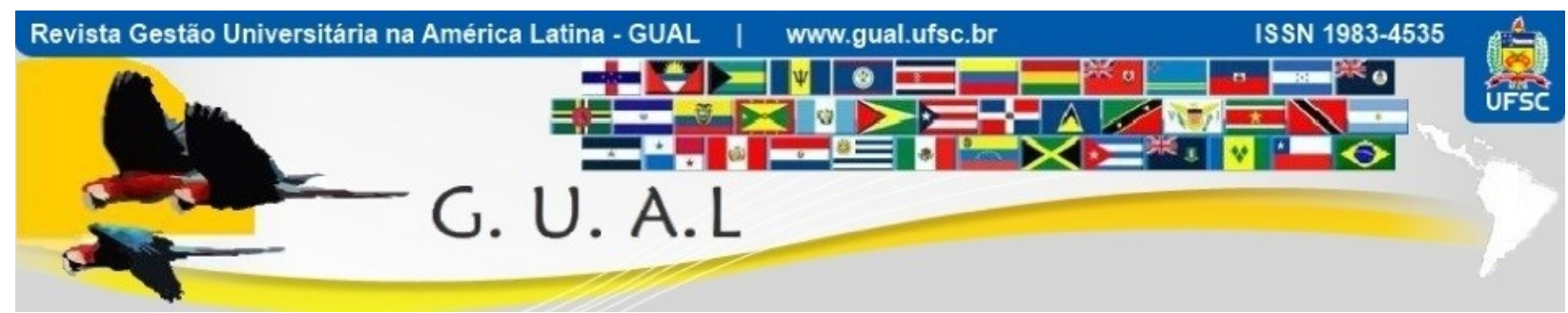

DOI: http://dx.doi.org/10.5007/1983-4535.2013v6n4p92

\title{
IMPLEMENTACIÓN DE LA CARRERA ACADÉMICA COMO MEDIO DE INTEGRACIÓN DEL NSPT A LA UNIVERSIDAD
}

\section{IMPLEMENTATION OF THE ACADEMI CAREER AS A WAY OF INTE-GRATING THE INSPT TO THE UNIVERSITY}

Noemi Susana Geromini, Mestre Universidad Tecnológica Nacional - UTN noegero@gmail.com

Carlos Gustavo Lovallo, Licenciado Universidad Tecnológica Nacional - UTN guslovallo@gmail.com

Recebido em 29/novembro/2013

Aprovado em 29/novembro/2013

Sistema de Avaliação: Double Blind Review

Esta obra está sob uma Licença Creative Commons Atribuição-Uso. 


\begin{abstract}
RESUMEN
Con el fin de mejorar la calidad académica de su cuerpo docente, el Instituto Nacional Superior del Profesorado Técnico -INSPT-, como Institución de Educación Superior no Universitaria, considera necesaria la planificación de estrategias que permitan generar espacios de pertenencia a sus docentes, que les otorguen estabilidad laboral y posibilidades de proyección, mediante el establecimiento de reglas claras e imparciales que protejan a la comunidad académica. La calidad académica se logra por medio de una clara definición de las características esperadas de los docentes y la excelencia se fomenta con procesos de evaluación continuos de todas las activida-des de la vida académica: enseñanza, investigación, extensión y gestión. La Carrera Académica, sistema que establece ingreso, permanencia y promoción de los docentes, resulta ser el recurso más adecuado para estimular la mejora continua de la tarea docente y lograr la excelencia académica, haciendo posible la integración de una institución no universitaria al sistema universitario. Mediante un exhaustivo análisis de la idiosincrasia de la comunidad educativa del INSPT, y aten-diendo a las características esperables de sus docentes, el Instituto procura adaptarse al régimen docente universitario vigente en la Universidad Tecnológica Nacional -UTN. Para lograr este objetivo, se han analizado el Estatuto Universitario, la Ordenanza 1182 del Consejo Superior y se ha elaborado un documento que, por un lado, fundamenta la necesidad de adecuar el régimen de ingreso, permanencia y promoción docente del INSPT a la Carrera Académica de la Universidad, y por otro, propone los pasos a seguir para su implementación, con el convencimiento de que su aplicación redundará en una continua mejora de la calidad académica del Instituto. El presente trabajo tiene como objetivos, por un lado, fundamentar y describir el proceso de ade-cuación del régimen docente del INSPT, a la Carrera Académica de la UTN, resaltando su valor como medio de integración del Instituto a la Universidad y a la vez, presentar una propuesta de im-plementación de la Carrera Académica al Instituto.
\end{abstract}

Palabras-clave: Carrera Académica. Calidad docente. Estabilidad docente. Integración a la Universidad.

\begin{abstract}
With the purpose of improving the academic quality of its teaching staff, the National Superior Ins-titute of the Technical Teacher Training College -INSTP-, as an non UniversityInstitution of Supe-rior Education, considers that a planning of strategies is required; this strategies shall allow the teachers to create spaces of belonging, which shall give them working stability and possibilities of projection, by means of the establishment of clear and non-partial rules which would protect the academic community. The academic quality is accomplished by a clear definition of the expected characteristics of the faculty and the excellence is encouraged by constant evaluating processes in every activity of the academic life: teaching, investigation, extension and paperwork. The Academic Career, a system which establishes joining, permanency and promotion of the teachers, turns out to be the most adequate resource to stimulate the unceasing improvement of the teaching work and accomplish the academic excelence, making it possible to integrate a non-university institution to the university system. Through an exhaustive analysis of the idiosyncrasy of the educational community of the INSPT, and attending to the expected characteristics of its teachers, the Institute is willing to adapt to the university teaching method which applies now at the National Technological University -UTN-. Looking forward to accomplish this, the following documents have been analyzed: the University Statute and the Ordinance 1182 of the Superior Board. After this, a paper was written, supporting the need to adaptthe system of joining, permanency and promotion of the teachers of the INSPT to the University Academic Career and also suggesting the steps that should be followed to its imple-mentation, having into account that its application will achieve a non-stopping improvement on the academic quality of the Institute. This paper has two main objectives. In the first place, describing and laying the foundations of the process of adaptation of the teaching regimen of the INSPT to the Academic Career of the UTN, emphasizing its value as a mean of integrating the Institute to the University. And, in the second place, submitting a proposal to implement the Academic Career to the Institute.
\end{abstract}

Keywords: Academic Career. Teaching quality.Teaching stability.Integration to the University. 


\section{FUNDAMENTACIÓN}

La legislación argentina que regula la educación superior, tanto universitaria como no universitaria, establece que el ingreso y permanencia del cuerpo docente en las instituciones debe ser realizado en un marco de autonomía académica, lo que les permite, a cada una de ellas, adecuarlo a las particularidades de la misma. Si bien no define la Carrera Académica, sugiere que su aplicación es necesaria para asegurar la calidad docente, basada en el mérito para el ingreso, promoción y permanencia de los docentes en la educación superior. Al tiempo que asegura, como derecho de los docentes, el acceso a la Carrera Académica, propone, como deber de éstos, la actualización y el perfeccionamiento que fije la misma.

La denominación de Carrera Académica hace referencia a un proceso que se contextualiza en un marco institucional determinado. Todas las instituciones exhiben una organización determinada que ordena su funcionamiento y los procesos que se van a desplegar en la misma.

El marco legal permite establecer parámetros de referencia, que contribuyen a fundamentar, por un lado, la necesidad de aplicación de la Carrera Académica, pero por otro lado, la libertad de cada institución para adecuarla según sus particularidades.

La ley de Educación Superior (LES), sancionada en el año 1995, menciona, entre otras atribuciones que configuran la autonomía universitaria, la de establecer el régimen de acceso, permanencia y promoción del personal docente y no docente y de designar y remover al personal.

Anteriormente a la LES, la Ley Federal de Educación (LFE), sancionada en el año 1993, ya establecía que el Estado Nacional debe fijar los lineamientos de la política educativa respetando, entre otros, el derecho de todos los docentes a la dignificación y jerarquización de su profesión y asegurando su capacitación para desempeñarse en cada uno de los niveles y modalidades del sistema educacional y su perfeccionamiento no sólo como docentes, sino también como investigadores y administradores educativos.

La LES establece que los objetivos de la Educación Superior, además de los mencionados por la LFE, son incrementar y diversificar las oportunidades de actualización y perfeccionamiento para los integrantes del sistema. Además, determina que las instituciones universitarias deben garantizar el perfeccionamiento de sus docentes, acorde con los requerimientos de la Carrera Académica. 
Continuando con la LES, en varios de sus artículos se mencionan elementos relacionados a la Carrera Académica, como el derecho de acceso a la misma mediante concursos públicos y abiertos de antecedentes y oposición y como el deber de perfeccionamiento en el marco de la misma.

Las normativas mencionadas se completan con otros documentos y resoluciones que organismos como el Ministerio de Educación formulan para su aplicación, con documentos dictados para instituciones en particular, y también con estatutos, reglamentos y disposiciones propios de cada Universidad o Instituto Superior no Universitario.

Si bien el marco normativo expuesto hasta aquí deja clara la pretensión de que se implemente la Carrera Académica en las instituciones de educación superior, también es cierto que la exigencia contempla sólo elementos esenciales, como los objetivos generales y las pautas fundamentales a cumplir, reservando la metodología y los instrumentos a emplear a la autonomía universitaria. De esta manera, cada institución de educación superior podrá incorporar aquellos elementos que permitan a través del desarrollo de la carrera académica el cumplimiento de sus finalidades institucionales.

La UTN aplica la Carrera Académica desde el ciclo lectivo 2005, con variados grados de avance en las distintas Unidades Académicas. Fueron varias las normativas que, desde ese momento reglamentaron su aplicación, hasta la sanción, en el año 2009, de la Ordenanza 1182, que es el marco regulatorio actual.

El INSPT, como Institución de Educación Superior no Universitaria, pretende iniciar el tránsito hacia la implementación de la Carrera Académica, con la finalidad de asegurar un proceso sostenido de construcción y mantenimiento de la calidad educativa y como medio de integración del Instituto a la Universidad. Para concretar este proceso, es preciso, por un lado, efectuar un estudio detallado del marco normativo vigente en la UTN y por el otro, realizar un profundo análisis de las particularidades del INSPT, de la realidad que presenta como Instituto Terciario no universitario, con el fin de identificar los mecanismos que le permitan adecuarse al régimen docente en vigencia en la UTN. Finalmente, se torna también necesario realizar un examen comparativo entre los actores y elementos (cargos docentes, dedicaciones, funciones, etc.) de cada uno de los dos escenarios, para adecuar los que son propios del INSPT, a la Carrera Académica, con el objetivo de sentar las bases para la definición de políticas y estrategias aptas para implementarla. Y que de esta forma, la Carrera Académica se convierta en una herramienta, quizás la más importante, para lograr la mejora continua de la 
tarea docente y la excelencia académica del INSPT, otorgando a sus docentes estabilidad laboral y posibilidades de proyección y colaborando en la evolución hacia una educación superior no universitaria equitativa, de calidad e integrada a la Universidad.

\section{ESCENARIOS}

\subsection{UNIVERSIDAD TECNOLÓGICA NACIONAL}

Desde el año 2004, la Carrera Académica estuvo regulada por laOrdenanza 1009 del Consejo Superior. En el año 2008, la Ordenanza 1009 fue derogada por la que rigeactualmente, la Ordenanza 1182, que adecuó y actualizó la Carrera Académica para el personal docente de la UTN.

El Estatuto Universitario sancionado en el año 2007 hace referencia a la Carrera Académica, presentándola como herramienta para la formación, evaluación y perfeccionamiento de los docentes.

Tanto en el período de transición como en la implementación en sí de la Carrera Académica, se fueron incorporando procedimentos generales que completaron el marco normativo.

Fue implementada por primera vez en el año 2005, y desde ese momento, se aplica a todos los docentes concursados, cada tres años, y a los docentes interinos, cada año.

\subsection{INSTITUTO NACIONAL SUPERIOR DEL PROFESORADO TÉCNICO}

El INSPT es una Institución de Educación Superior no universitaria que fue creado en 1959 por Decreto $N^{\circ} 15.958$ con el objetivo de formar, capacitar y perfeccionar profesores para la educación técnica. Hasta el año 1995 dependió del Consejo Nacional de Educación Técnica (CONET), cuando este organismo se disolvió, por decreto 606/95, el Instituto fue transferido a la UTN.

Actualmente el INSPT forma:

- Técnicos Superiores (Primer ciclo de tres años) para desempeñarse en áreas tecnológicas.

- Profesores para la Educación en los niveles medio, técnico y superior no universitario y en empresas. (Segundo ciclo/ cuartoaño).

Algunos de los objetivos del INSPT son: 
- Formar profesores con competencias académicas tecnológicas y sociales y capacidad de desarrollo de valores para asegurar una educación tecnológica de calidad.

- Asegurar la formación de Técnicos Superiores en Ciencias y Tecnologías.

- Fortalecer las áreas de docencia e investigación y actuar como centro de investigación y experimentación en los campos de tecnología y educación.

- Transferir tecnología a la comunidad a través de la docencia, la investigación y consultoría.

- Organizar y participar enseminarios y congresos especializados de carácter nacional e internacional.

Por ser un instituto terciario dependiente de laUniversidad, algunos de sus cargos docentes sonterciarios y otros son universitarios. El cuerpo docente del INSPT está formado por profesores, jefes de trabajos prácticos, ayudantes de primera y ayudantes de segunda (éstos últimos alumnos).

Hasta el año 2009, algunos de sus cargos docentes revestían la categoría de titular, gracias a la sanción de la Ley Nacional 24.129, que en 1992 titularizó a los docentes dependientes del Ministerio de Cultura y Educación y del CONET, y más tarde a las Disposiciones 1759/07 y 1760/07, ambas del Director del INSPT, Ing. Héctor René Gonzalez.

En el año 2009, mediante la Disposición1443/09, se implementaron los primeros concursos docentes para profesores en el INSPT. En el año 2011 se realizaron nuevamente concursos docentes, los últimos hasta el día de hoy. En ambas instancias, los cargos concursados adquirieron la categoría de titulares por siete años, por Disposición del Director.

El mecanismo que rige actualmente para la designación de docentes es el siguiente: El Consejo de Carrera , formado por tres docentes titulares (tres profesores de la Carrera o bien dos profesores de la Carrera y un docente del Laboratorio correspondiente) y un docente suplente, luego de analizar y evaluar los antecedentes académicos de los docentes que se postulan, propone al Director de Carrera al docente elegido para cubrir determinada instancia curricular (asignatura) y/o cargo de Laboratorio. Luego de esta instancia, el Director de Carrera, en función de lo propuesto por el Consejo de Carrera, eleva a la Coordinación Académica la propuesta de designación del nuevo docente. A continuación, la Coordinación Académica la eleva al Secretario Académico y finalmente, el Director del Instituto, en caso de conformidad, realiza la designación definitiva. 
En virtud de lo expuesto, actualmente en el INSPT, aproximadamente el $60 \%$ de sus cargos de profesores reviste caracter de titular, y de sus cargos de auxiliares docentes, sólamente el $20 \%$.

Ante esta realidad académica del INSPT, es clara la necesidad de implementar la Carrera Académica para asegurar la estabilidad a los docentes en los cargos ganados por concurso, y al mismo tiempo, garantizar la calidad académica de éstos.

Más adelante en este trabajo, se presentará una propuesta de construcción e implementación de la Carrera Académica para el Instituto.

\section{ANÁLISIS COMPARATIVO}

En este punto es pertinente realizar una comparación de los elementos esenciales de la Carrera Académica en ambos escenarios.

Se exhibe en la tabla 1, un paralelo entre diversos elementos relacionados con la Carrera Académica, de manera que puedan entenderse cuáles son los aspectos a tener en cuenta en el proceso de adecuación del INSPT a la normativa vigente en la UTN.

Una vez expuestos los escenarios, es decir, la Universidad de la cual proviene el marco normativo general, y el Instituto donde se pretende generar los mecanismos para adecuarse a dicha normativa, y exhibido el paralelo entre los elementos significativos de cada uno de los escenarios, será el momento de realizar una conceptualización de la Carrera Académica, tal como se la proyecta en el INSPT.

\begin{tabular}{|c|c|c|}
\hline & U.T.N. & I.N.S.P.T. \\
\hline & $\begin{array}{l}\text { - ComisiónEvaluadora. } \\
\text { - ConsejoDirectivo. } \\
\text { - Consejo Superior. }\end{array}$ & $\begin{array}{l}\checkmark \text { ComisiónEvaluadora. } \\
\checkmark \text { Director. }\end{array}$ \\
\hline $\begin{array}{l}\text { Organización / } \\
\text { Autoridad }\end{array}$ & $\begin{array}{l}\text { Departamentos } \\
\text { Director de departamento. }\end{array}$ & $\begin{array}{l}\checkmark \text { Carreras } \\
\checkmark \text { Director de Carrera. }\end{array}$ \\
\hline $\begin{array}{l}\text { Categorías } \\
\text { docentes }\end{array}$ & 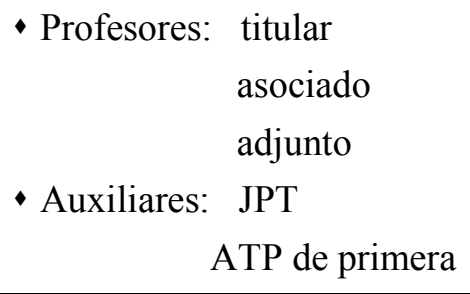 & $\begin{array}{l}\checkmark \text { Profesores } \\
\checkmark \text { Auxiliares: JTP } \\
\text { ATP de primera }\end{array}$ \\
\hline Sit. revista & Titular - Interino - Suplente & Titular - Interino - Suplente \\
\hline Tipo de cargo & Universitarios & Terciarios y Universitarios \\
\hline
\end{tabular}




\begin{tabular}{|c|c|c|}
\hline Dedicaciones & exclusiva - semiexclusiva - simple & Horascátedra - dedicaciónsimple \\
\hline Funciones & $\begin{array}{l}\text { 1. Docencia } \\
\text { 2. Investigación y desarrollo } \\
\text { 3. Formación } \\
\text { 4. Extensión } \\
\text { 5. GestiónAcadémica y de } \\
\text { Gobierno }\end{array}$ & $\begin{array}{l}\text { 1. Docencia } \\
\text { 2. Investigación y desarrollo } \\
\text { 3. Formación } \\
\text { 4. Extensión } \\
\text { 5. GestiónAcadémica y de Gobierno }\end{array}$ \\
\hline
\end{tabular}

Tabla 1 Cuadro comparativo entre elementos de UTN y INSPT

\section{CONCEPTUALIZACIÓN}

Según definición de UTN, la Carrera Académica es el sistema mediante el cual se establece el ingreso, permanencia y promoción de los docentes de la Universidad.

Proyectada en el INSPT, la Carrera Académica puede definirse como el proceso que el trabajador docente transita desde que ingresa al INSPT en tal carácter, hasta su retiro, incluyendo la reglamentación de los aspectos involucrados académicos, laborales e institucionales que hacen a su actividad.

El propósito central es contribuir al mejoramiento continuo de las actividades inherentes a la institución, impulsando el desarrollo de las potencialidades del cuerpo docente, mejorando las condiciones en que realiza su trabajo y reconociendo sus logros, en un proceso evolutivo hacia la superación permanente en la enseñanza, la investigación y la extensión; con el fin último de alcanzar la integración con la UTN.

La implementación de la Carrera Académica permitirá, por un lado, la evaluación sistémica del conjunto de los docentes, ya que contempla a la evaluación como un instrumento pedagógico y de gestión, y por el otro, el diseño de planes de mejora a través de la detección de fortalezas y debilidades. Esto implica que en el proceso se deberá involucrar a todos los docentes y abarcar todas las actividades de la vida institucional, es decir, enseñanza, investigación, extensión y gestión, impulsando la mejora de la función docencia.

Mediante la evaluación se procura obtener información rigurosa y confiable sobre diversos aspectos de la labor del docente, que luego serán valorados por diferentes actores. Así, mediante el cruce de la información procedente de distintas fuentes, se obtendrá una matriz del desenvolvimiento del docente, de su formación, de su cumplimiento y de sus logros. 
Las posibilidades de implementación de la Carrera Académica en el INSPT suponen ineludiblemente la existencia, a nivel de gestión, de un cuidadoso planeamiento académico institucional, que defina los parámetros a considerar para la conformación de la planta docente, su estructuración y su permanencia.

Este planeamiento académico definirá, a partir de las necesidades emergentes de los ciclos de las diferentes tecnicaturas y profesorados, del número de asignaturas y las características de ellas, de la forma de dictado, del número de alumnos que cursan las asignaturas y de la cantidad máxima de asignaturas por docente, la metodología adecuada para implementar la Carrera Académica, de acuerdo con las particularidades del INSPT, no presentes en la Universidad.

Esencialmente, el planeamiento académico debería contemplar diversos mecanismos que permitan, en primera instancia, la formación y selección de recursos humanos para el ingreso al sistema. También debería permitir la determinación de las condiciones a las que se encuentra sujeta, una vez logrado el ingreso del docente, su permanencia, perfeccionamiento y promoción. Por último, debería establecer las pautas y procedimientos para la salida del sistema.

Tal como se mencionó anteriormente, en la UTN, actualmente la Carrera Académica está regulada por la Ordenanza 1182/08. Previo a la presentación de la propuesta de implementación en el INSPT, se realiza una breve descripción de la mencionada Ordenanza.

\subsection{ORDENANZA 1182/08}

Los aspectos esenciales pueden resumirse en los siguientes puntos:

- El ingreso y la modificación de la jerarquía académica, se efectúan por concurso abierto y público de títulos, antecedentes y oposición, según las reglamentaciones en vigencia. El perfeccionamiento docente es continuo e institucional. La permanencia es conforme con los resultados de la evaluación.

- Las categorías establecidas para los docentes son: profesores (titular, asociado, adjunto) y auxiliares (jefes de trabajos prácticos, ayudantes de trabajos prácticos de primera).

- La actividad académica comprende el desarrollo de las siguientes funciones: Docencia, Investigación y Desarrollo, Formación, Extensión, Gestión Académica y de Gobierno.

- El régimen de dedicaciones es el siguiente: Dedicación simple, dedicación semiexclusiva y dedicación exclusiva.

- La permanencia depende del resultado de las instancias de evaluación previstas. Los docentes concursados que obtienen resultados positivos, renuevan su condición por un 
nuevo período. En caso de obtener más de una evaluación negativa, vencido el período de designación, se llama a nuevo concurso .

- La evaluación del docente comprende tres módulos, ellos son: Cumplimiento del plan de actividades académicas, cumplimiento de las obligaciones conexas a las actividades académicas y desempeño frente a alumnos.

- La Universidad se compromete a promover y facilitar actividades de actualización y perfeccionamiento para sus docentes.

- La evaluación de la actividad académica se realiza mediante la autoevaluación de los docentes y los informes de sus superiores académicos. Las actividades de investigación y desarrollo son evaluadas por pares. La evaluación de las actividades conexas se realiza mediante informes de los sectores correspondientes. El desempeño frente a alumnos es evaluado mediante encuestas a los alumnos regulares.

- El Director de Departamento es quien inicia el Proceso de Evaluación, y todos los informes recabados son evaluados por la Comisión de Evaluación, para finalizar el proceso emitiendo un dictamen sobre el docente evaluado.

- El resultado de las calificaciones puede ser: Insuficiente, Suficiente, Muy bueno, Sobresaliente. La calificación mínima para un dictamen favorable es Suficiente.

- La Comisión de Evaluación está formada por tres profesores de Universidades Nacionales, designados por el Consejo Directivo.

- El dictamen de la Comisión de Evaluación es elevado al Consejo Directivo. Éste, a su vez, eleva la propuesta de designación del docente por un nuevo período (en caso de dictamen favorable) al Consejo Superior, que es el facultado para realizar las designaciones docentes.

\subsection{PROPUESTA DE CARRERA ACADÉMICA PARA EL INSPT}

Se presenta a continuación una propuesta de construcción e implementación de la Carrera Académica en el Instituto.

Se analizan uno a uno, cuáles son los capítulos de la Ordenanza 1182 que pueden aplicarse tal como están y cuáles los que, por las particularidades del INSPT, para implementarlos requieren ser modificados.

Con respecto al Capítulo I, Elementos Básicos, como ya se mencionó anteriormente en este trabajo, el INSPT implementó, en el año 2009, los primeros concursos docentes. Este hecho confirma la convicción del Instituto respecto de la implementación de concursos como método para el ingreso y la modificación de la jerarquía académica.

Desde el momento en que se produce una vacante, el concurso propiamente dicho abarca varias etapas: el Director del Instituto llama a concurso, el jurado expresamente convocado realiza una evaluación de los antecedentes de los aspirantes, los aspirantes 
aceptados realizan una prueba de oposición, que consiste en una clase y un coloquio sobre el plan de trabajo presentado. El Jurado, luego de una evaluación integral, se expide presentando la nómina de concursantes por orden de mérito. El concurso de da por finalizado cuando el profesor es designado por el Director, ad-referéndum del Rector, por un período de siete años.

En cuanto al Capítulo II, Categorías y Niveles Jerárquicos, las particularidades del INSPT impiden que la Ordenanza 1182 pueda aplicarse exactamente igual que en la Universidad, aunque sí podría llevarse a cabo con algunas modificaciones.

El INSPT establece las categorías de Profesor y de Auxiliar Docente, ésta última incluye Jefe de Trabajos Prácticos y Ayudante de Trabajos Prácticos. Si bien no contempla niveles jerárquicos como en la Universidad, sí existen, encuadradas como situación de revista, las figuras de Titular e Interino, para cada uno de los cargos mencionados.

Los requisitos para acceder a las mencionadas categorías son los establecidos en el Anexo II de la Disposición 1443/09 del INSPT, que reglamenta los concursos docentes, y también por las disposiciones en vigencia. La función básica del profesor es dictar las clases teórico-prácticas de la asignatura. La de los auxiliares docentes consiste, bien en trabajar en la clase colaborando con el profesor del curso, bien desempeñarse en los laboratorios del Instituto.

Por ser un Instituto Terciario transferido del CONET a la UTN, los cargos no son en su totalidad universitarios, los cargos de profesores mantienen su carácter de cargo terciario como herencia del CONET, y para los de auxiliares docentes se combinan los cargos terciarios reservados a los reemplazos, con cargos universitarios destinados a los de creación.

Tampoco se aplican las dedicaciones vigentes en la Universidad (simple, semiexclusiva y exclusiva), en el INSPT las dedicaciones son de horas cátedra para los profesores y de dedicaciones simples para los auxiliares.

La actividad académica del INSPT, al igual que en la Universidad, comprende el desarrollo de las siguientes funciones:

a)Docencia: De acuerdo con el Anexo II de la Disposición 1443/09 del INSPT, las más importantes son: dictar las clases teórico-prácticas de la asignatura, conducir la enseñanza de acuerdo con los planes y normas establecidos por el INSPT, resolver y evaluar el plan de enseñanza y metodología en coordinación con las otras asignaturas de la Carrera a la que pertenezca, distribuir las tareas a cumplir por los ayudantes de $\mathrm{TP}$, participar en reuniones y en actividades de la Carrera según el plan propuesto por el Director de Carrera. En la función docencia, la Ordenanza 1182 de la UTN puede aplicarse sin modificaciones en el INSPT. 
Investigación y Desarrollo: Esta función se relaciona con la elaboración y ejecución de trabajos, proyectos, informes y publicaciones que impliquen un avance en el conocimiento de una determinada temática. Quizás sea esta la función que mayor dificultad presente en el momento de aplicar la Ordenanza 1182 de la UTN al INSPT, por varias razones. Por un lado, al carecer de dedicaciones semiexclusiva y exclusiva, los docentes reciben su remuneración acorde con la cantidad de horas clase que dictan de una determindad asignatura. Esto implica que, para realizar cualquier actividad que no sea meramente el dictado de clase, deban dedicar tiempo que no está previsto en su remuneración. Por otro lado, el Programa de Incentivos del Ministerio de Educación no es aplicable a docentes terciarios, motivo por el cual, los profesores del INSPT que no se desempeñan, además, en una Universidad, no perciben el incentivo a docente-investigador, en el marco del Programa Nacional. La realidad indica que en el Instituto, la mayoría de los docentes que se dedican a la investigación, lo hace en el marco de otra Institución, generalmente universitaria, en la cual se desempeñan. Finalmente, y no menos importante es el hecho de que el cuerpo docente del INSPT proviene, en su mayoría, de la carrera de Profesorado, a diferencia del docente que se desempeña en la Universidad, que proviene de una carrera de grado de la misma u otra Universidad. Esta situación se refleja en el hecho de que no todos los profesores del Instituto tienen la vocación y la competencia necesarias para realizar investigación, y esta realidad no desmerece su eficiencia y calidad como profesor.

Con lo expuesto, queda claro que en este aspecto, la aplicación directa de la Ordenanza 1182 se torna prácticamente insostenible en el INSPT. Esta propuesta contempla como posibilidad de adecuación, para subsanar al menos dos de las tres obstáculos mencionados, complementar el cargo de profesor de horas-clase con un cargo auxiliar, de manera que se amplíe el tiempo disponible del docente para realizar investigación.

b)Formación: En este punto, lo que se espera de un docente del INSPT no escapa a las exigencias de actualización y perfeccionamiento aplicables a todos los docentes en general.

c)Extensión: Esta función contempla el desarrollo de actividades y programas relacionados con las asignaturas, carreras y laboratorios, la realización de actividades de asistencia comunitaria, y en general, todo tipo de trabajo que implique transferencia de conocimientos hacia adentro y hacia afuera de la Universidad.

d)Gestión Académica y de Gobierno: Esta función abarca las misiones de responsabilidad institucional, como ser la asistencia a reuniones de Carrera, la presentación de 
informes o documentación solicitada por autoridad competente, la integración de jurados y/o comisiones evaluadoras.

En estas tres últimas funciones es directamente aplicable al INSPT, la Ordenanza 1182 de la UTN.

Considerando el Capítulo III, Ingreso y Permanencia, las pautas fijadas en la Ordenanza 1182 pueden aplicarse directamente al cuerpo docente del INSPT, tanto en el ingreso, ya mencionado en este trabajo, como en la permanencia de los docentes. En cuanto a la permanencia, los docentes concursados que durante la Carrera Académica obtuvieran resultados positivos en las instancias de evaluación, renovarán su condición por un nuevo período. En caso de registrar más de una evaluación negativa, vencido el período de designación, se llamará a un nuevo concurso.

Con respecto al Capítulo IV, Evaluación, esta propuesta contempla la aplicación directa de la Ordenanza 1182. La evaluación del docente comprende tres módulos:

1. Cumplimiento del plan de actividades académicas: Abarca las funciones a., b., c. y d., de la actividad académica mencionadas anteriormente.

2. Cumplimiento de las obligaciones conexas a las actividades académicas: Se refiere a las tareas propias de la función e.

3. Desempeño frente a alumnos: Para evaluar este ítem se tendrán en cuenta, para cada docente, la opinión de sus alumnos, la autoevaluación del docente y el informe de su superior académico.

En relación con el Capítulo V, Formación y Perfeccionamiento Docente, el INSPT, por medio de sus autoridades, decidirá si se llevarán a cabo acciones que promuevan y faciliten las actividades de actualización y perfeccionamiento pedagógico y disciplinar de sus docentes, y en caso afirmativo, cuáles serían las vías para lograrlo. Esto significa que este capítulo de la Ordenanza 1182 se aplicará o no, en función de futuras decisiones institucionales.

El Capítulo VI, Reglamento de Evaluación del Docente, trata la forma de evaluar al docente. Por un lado, las actividades académicas: Las actividades de docencia serán evaluadas mediante los informes que presente, tanto el mismo docente, como también el Director de Carrera. Las actividades de investigación y desarrollo serán evaluadas por pares. Y en lo relativo a las actividades de formación, los docentes deberán presentar las constancias correspondientes, que avalen dichas tareas. Por otro lado, para evaluar el cumplimiento de las 
obligaciones conexas, el Director de Carrera solicitaría a los sectores involucrados, un informe del docente. Y por último, el desempeño frente a los alumnos se evaluará solicitando a los alumnos su opinión acerca del docente, mediante el método de encuestas de identificación indirecta.

El proceso principal de evaluación de toda la documentación completa del docente será llevado a cabo por una Comisión Evaluadora, conformada a tal fin.

Con respecto a la Comisión Evaluadora, este trabajo propone, para su conformación, similar mecanismo que el contemplado para la creación de los Consejos de Carrera. La Comisión Evaluadora estará formada por el Director de Carrera, como presidente, y tres profesores de la Carrera. Contará además, con un profesor de la Carrera, en carácter de miembro suplente.

La Comisión Evaluadora analizará toda la documentación recibida del docente evaluado y, luego de realizar una entrevista personal al docente, se expedirá sobre el desempeño de este último, emitiendo el correspondiente dictamen, debidamente fundamentado y firmado por todos sus miembros.

El dictamen de la Comisión Evaluadora será considerado por el Secretario Académico y luego por el Director del Instituto. En caso de conformidad con el dictamen, el nuevo docente será designado por el Director del Instituto ad-referendum del rector de la UTN, por un nuevo período.

En cuanto al Capítulo VII, Las Calificaciones, la Ordenanza 1182 puede aplicarse directamente al INSPT, estableciendo, al igual que en la UTN, la siguiente tabla de calificaciones:

- Insuficiente.

- Suficiente.

- Muy Bueno.

- Sobresaliente.

La calificación mínima requerida para que el dictamen sea favorable es la de Suficiente.

Se ha realizado hasta aquí una breve descripción y análisis de las condiciones favorables y las desfavorables que presenta el INSPT a la hora de intentar aplicar cada uno de los capítulos de la Ordenanza 1182 de la UTN. El análisis realizado permitió proponer pautas para favorecer la aplicación de dicha Ordenanza al Instituto. 


\section{CONCLUSIONES}

Acorde con los objetivos planteados en el presente trabajo, se ha presentado una propuesta de aplicación de la Carrera Académica al Instituto. Esta propuesta es sólo un esbozo del proceso que podría llevarse a cabo para lograr la implementación de la Carrera Académica en el INSPT. Es un conjunto de pautas deducidas de un análisis de la realidad, pasible de modificaciones y mejoras que optimicen el resultado.

Queda claro que este proceso, en tanto proyecto institucional, se debería completar, por un lado, con políticas adecuadas que, desde la Dirección del Instituto y desde la Secretaría Académica, aseguren la infraestructura, los mecanismos y los recursos necesarios para efectivizarlo, y por el otro, con un compromiso académico y social de los docentes involucrados y de toda la comunidad educativa del INSPT.

En la medida en que el INSPT continúe, como hasta ahora, con la realización de los concursos docentes para modificar la jerarquía de sus actuales docentes en actividad, los amplíe para gestionar el ingreso de los docentes necesarios para cubrir cargos vacantes, y logre implementar la Carrera Académica, estará realmente transitando la integración definitiva a la Universidad.

\section{BIBLIOGRAFÍA}

Argentina. Ley Federal de Educación, Ley N² 24.195/93.Boletín Oficial de la República Argentina, de 5 de mayo, núm. 27.632. Disponible en: http://ar.vlex.com/source/bora1675/issue /1993/5/5/1

Argentina. Ley de Educación Superior, Ley N²4.521/95. Boletín Oficial de la República Argentina, de 10 de agosto, núm. 28.204. Disponible en: http://www.me.gov.ar/ consejo/cf ley superior.html

Argentina. Instituto Nacional Superior del Profesorado Técnico. Disposición N 1443/09. Reglamento de Concursos para la designación de profesores en el Instituto Nacional Superior del Profesorado Técnico.

Argentina. Universidad Tecnológica Nacional. Sec. Académica y Sec. de Planeamiento, "Sistema de Evaluación - Carrera Académica", 2011.

Gonzalez, J.M. (2004). Carrera Académica, Una Alternativa Posible? Universidad de la Cuenca del Plata. Corrientes. Argentina. Disponible en http://www.inpeau.ufsc.br/coloquio04/completos/Jos \%E9\%20Mar\%EDa\%20Gonz\%E1lez\%20-\%20Carrera\%20acad\%E9mica.doc 